\title{
Raltegravir IN Treatment NaÏve Patients
}

\author{
F. Cossarini ${ }^{1}$, A. Castagna ${ }^{1}$, A.Lazzarin ${ }^{1,2}$ \\ ${ }^{1}$ Department of Infectious Diseases, San Raffaele Scientific Institute, Milan, Italy, \\ ${ }^{2}$ Vita-Salute San Raffaele University, Milan Italy
}

\begin{abstract}
Raltegravir is the first integrase inhibitor approved for the treatment of HIV infection based on the superior efficacy it showed compared to optimized backbone therapy alone in patients harboring multidrug resistant viruses. Studies on naïve patients showed comparable efficacy of raltegravir and efavirenz and just recently the US Food and Drug Administration (FDA) approved raltegravir for the use in naïve patients based on the favorable results of the international double-blind phase III STARTMRK trial. Additional interesting findings were the faster, and not yet explained, decay of HIV-1 RNA and the higher CD4+ cells increase in the raltegravir group as compared to the efavirenz group.

Raltegravir is generally well tolerated and adverse events were generally similar in raltegravir and comparator arms throughout all studies. When compared to efavirenz, patients on raltegravir showed less incidence of central nervous system-related adverse events. In studies on experienced patients higher incidence of cancers was found in the raltegravir arm: a relationship with the drug was, however not confirmed in a recent review considering all raltegravir studies. Raltegravir also showed a safe lipid profile expecially in naive patients, finding that renders the drug attractive for patients with other cardiovascular risk factors.

All this characteristics in association with its specific mechanism of action, make raltegravir an interesting drug for naïve patients and a large use in this type of patients is predictable. Only time and experience, however, will tell us whether raltegravir will maintain its promises in the long run.
\end{abstract}

\section{INTRODUCTION}

Efficacy of antiretroviral treatment (HAART) has dramatically reduced progression to AIDS and death in HIV infected individuals [1,2] although this treatment is still not available in many areas of the world especially where the pandemic has the higher prevalence. The absence of a realistic vaccine or eradication strategy in the near future renders chronic antiretroviral therapy the sole foreseeable therapy for HIV infection for years to come $[3,4]$. Development of drug resistance on therapy, as well as acquisition of a resistant viral strain at the time of infection reduce the option of treatment, a crucial issue for first line regimen too $[5,6]$. Current guidelines recommend initiation of an- tiretroviral treatment with two nucleoside reverse transcriptase inhibitors (NRTIs) in association with either an NNRTI (efavirenz being the preferred) or a boosted PI (lopinavir/ritonavir or atazanvir/ritonavir) [7, 8]. Short- and long-term toxicities of antiretrovirals have an additional impact on adherence to treatment and its long-term success $[9,10]$. All these issues underline the continuous need for new and better tolerated antiretroviral compounds. In the past few years several new agents have become available on the market: new protease inhibitor (PI) darunavir showed activity against PI-resistant viral strains and the new non-nucleoside reverse transcriptase inhibitor (NNRTI) etravirine [11] is approved by the US Food and Drug Administration (FDA). Perhaps even more importantly, new classes of drugs, targeting different steps in the HIV life cycle, were licensed: the CCR5 co-receptor inhibitor maraviroc [12] and the integrase inhibitor raltegravir [13]. Recently, agents approved for the use in experienced patients have been studied in naive patients too, thanks to their efficacy and tolerability profiles and this have happened for new drug classes as well.

This review summarizes the characteristics of raltegravir, the first integrase inhibitor approved for HIV infection, focusing on its use on naïve patients. Although not yet recommended by international guidelines, just recently the US FDA approved raltegravir for the use in naïve patients based on the favorable results of the STARTMRK trial [14]. Its mechanism of action, efficacy and tolerability profile, make raltegravir a very interesting agent for first-line antiretroviral treatment.

\section{EFFICACY}

Raltegravir is the first integrase inhibitor approved for the treatment of HIV infection based on the superior efficacy it showed compared to optimized backbone therapy alone in patients harboring multidrug resistant viruses [13]. Due to the favorable profile in terms of efficacy and tolerability, studies on naïve patients began right after. The first clinical trial on naïve patients was Protocol 004, a multicenter, randomized, doubleblind, placebo-controlled dose-ranging phase II study which tested the efficacy of raltegravir at four different doses (100, 200, 400 and $600 \mathrm{mg}$ twice daily), in association with two NRTIs [15]. In the first part of the trial 35 patients were randomized to receive either 
placebo $(\mathrm{n}=7)$ or raltegravir $(\mathrm{n}=28)$ at one of the four dosages as monotherapy for four days [16]. Patients were stratified by HIV-RNA levels $\leq$ or $>50000$ copies/ml; mean HIV-RNA was 4.53 to 4.97 Log10 copies/ml and CD4+ cell counts were 256 to 569 cell $/ \mu \mathrm{L}$. After 10 days of treatment mean HIV-RNA reduction in the combined raltegravir groups was 2.0 $\log _{10}$ copies $/ \mathrm{ml}$ from baseline, significantly greater than what observed in the placebo arm $(p<0.001$ for the comparison with each raltegravir arm). 50\%-70\% of patients in the raltegravir arm reached HIV$\mathrm{RNA}<400$ copies/ml by day 10 as compared to none in the placebo arm and at least one patient in each raltegravir arm reached HIV-RNA $<50$ copies/ml as compared to none in the placebo group. The study then continued in a second part where patients on raltegravir continued on their dosage schedule with the addition of tenofovir and lamivudine, and patients on the placebo arm started efavirenz also in association with tenofovir and lamivudine. Additional 171 patietns were randomized to receive raltegravir or efavirenz in the second part of Protocol 004, leading to a total of 198 treated patients ( 5 patient from part I did not continued to part II and 2 randomized patients were not treated) [15]. Results from this 48 weeks study showed no difference in efficacy between raltegravir arms, and although the trial was not powered for efficacy comparison with the standard of care, when raltegravir groups were combined in one group only, there was no difference in response between raltegravir and efavirenz based regimens. After 48 weeks of treatment $86 \%(137 / 160)$ of patients in the raltegravir arm and $87 \%(33 / 38)$ of patients in the efavirenz arm had HIV1 RNA $<50$ copies $/ \mathrm{ml}$. These results were confirmed at 96 weeks [17] and recently at 144 weeks: $80 \%$ of patients in the raltegravir arm and $76 \%$ of patients in the efavirenz arm had HIV-1 RNA < 50 copies/ml [18].

STARTMRK is the ongoing phase III trial comparing the efficacy and tolerability of raltegravir in comparison to efavirenz, both in association with tenofovir and emtricitabine. The 48 weeks results of this international, double-blind, non inferiority trial have just been reported [14]. Patients were enrolled between September 2006 and June 2008 if they had HIV-1 RNA levels
$>5000$ copies $/ \mathrm{ml}$, were previously naïve, not pregnant nor breastfeeding and had liver enzymes less than 5 times the upper limit of normal in case of viral hepatitis co-infection. In addition, patients had to have an HIV genotype at baseline showing sensitivity to efavirenz, tenofovir and emtricitabine, resistance to raltegravir was not tested. There was not CD4+ cell limit, and patients were stratified by baseline HIV-RNA $(>50000$ vs $\leq 50000)$ and viral hepatitis co-infection status. Patients were randomized in a $1: 1$ ratio to receive either raltegravir at the dose of $400 \mathrm{mg}$ twice daily or efavirenz at the standard dose of $600 \mathrm{mg}$ once daily. The primary end point of the study was achievement of HIV-RNA $<50$ copies/ml at week 48 and primary analysis were performed with a non-completer $=$ failure approach. Secondary endpoints were the achievement of HIV-RNA $<400$ copies $/ \mathrm{ml}$ and the change from baseline CD4+ cell count.

The study enrolled 566 patients, 282 were assigned to the raltegravir arm and 284 to the efavirenz arm. 257 and 247 patients completed at least 48 weeks of study in the raltegravir and efavirenz arm respectively.

In primary end-point analysis the rate of achievement of HIV-RNA $<50$ copies/ml at Week 48 was $86 \%(\mathrm{n}=241)$ patients in the raltegravir arm and $82 \%$ $(\mathrm{n}=230)$ patients in the efavirenz arm, demonstrating non-inferior efficacy of raltegravir when compared to efavirenz (Fig. 1). Compared to patients on efavirenz, those in the raltegravir arm had also a slightly but significant greater mean increase in CD4+ cell count at Week 48 compared to baseline: 189 cells $/ \mu \mathrm{L}$ vs 163 cells $/ \mu \mathrm{L}(\mathrm{p}=0.02)$ (Fig. 3).

An interesting observation, reported in the phase II trials and confirmed in this study, was the shorter time to virologic suppression for patients in the raltegravir arm compared to efavirenz arm (log-rank test $\mathrm{p}<0.0001)$. Mathematical models explained this phenomenon with a shorter phase II in viral decay in patients treated with raltegravir; the authors suggested that this raltegravir effect might have an impact in reducing the time needed for clearance of infection [19]. Although the possibility of viral clearance after infection might be still unrealistic, the additional specific effect of integrase inhibition on viral decay might have

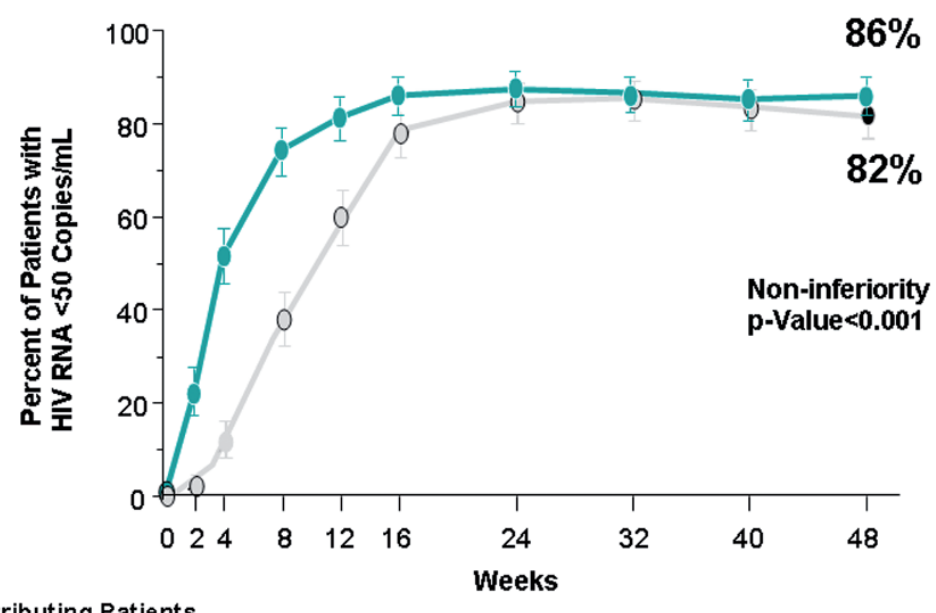

Number of Contributing Patients

O Raltegravir 400 mg b.i.d. * 281279281279281

O Efavirenz 600 mg q.h.s. * $282282 \quad 282 \quad 282 \quad 281$
281
Fig. 1. Proportion of Virologic Success $(\mathrm{HIV}-1 \mathrm{RNA}<50$ copies $/ \mathrm{ml}$ ) in raltegravir and efavirenz arms (From Lennox JL et al. Lancet 2009). 


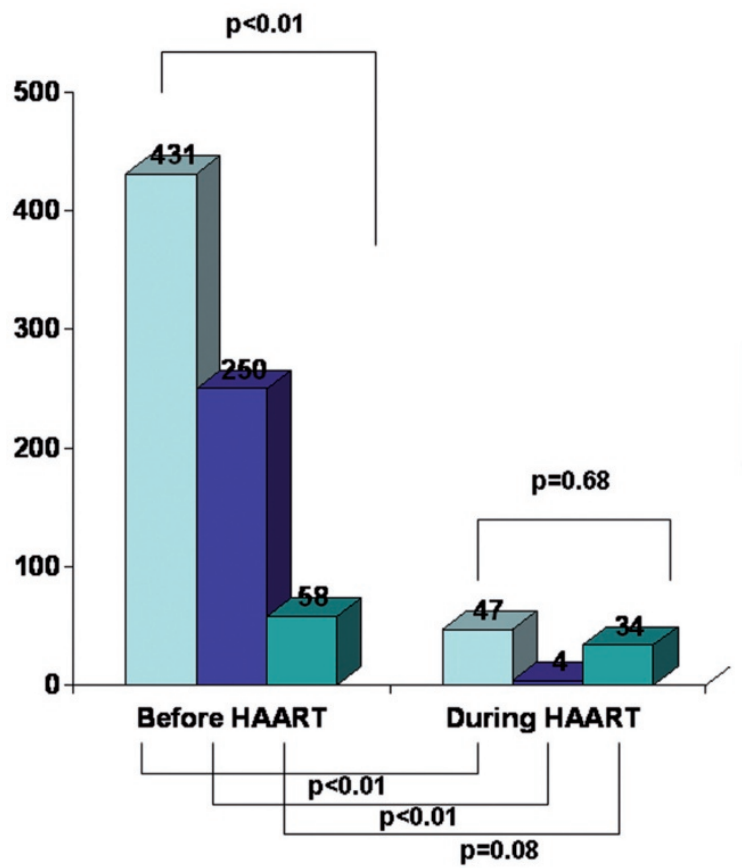

Fig. 2. Decrease in HIV-1 DNA during antiretroviral treatment (From Koelsch,K.K. et al. JID 2008).

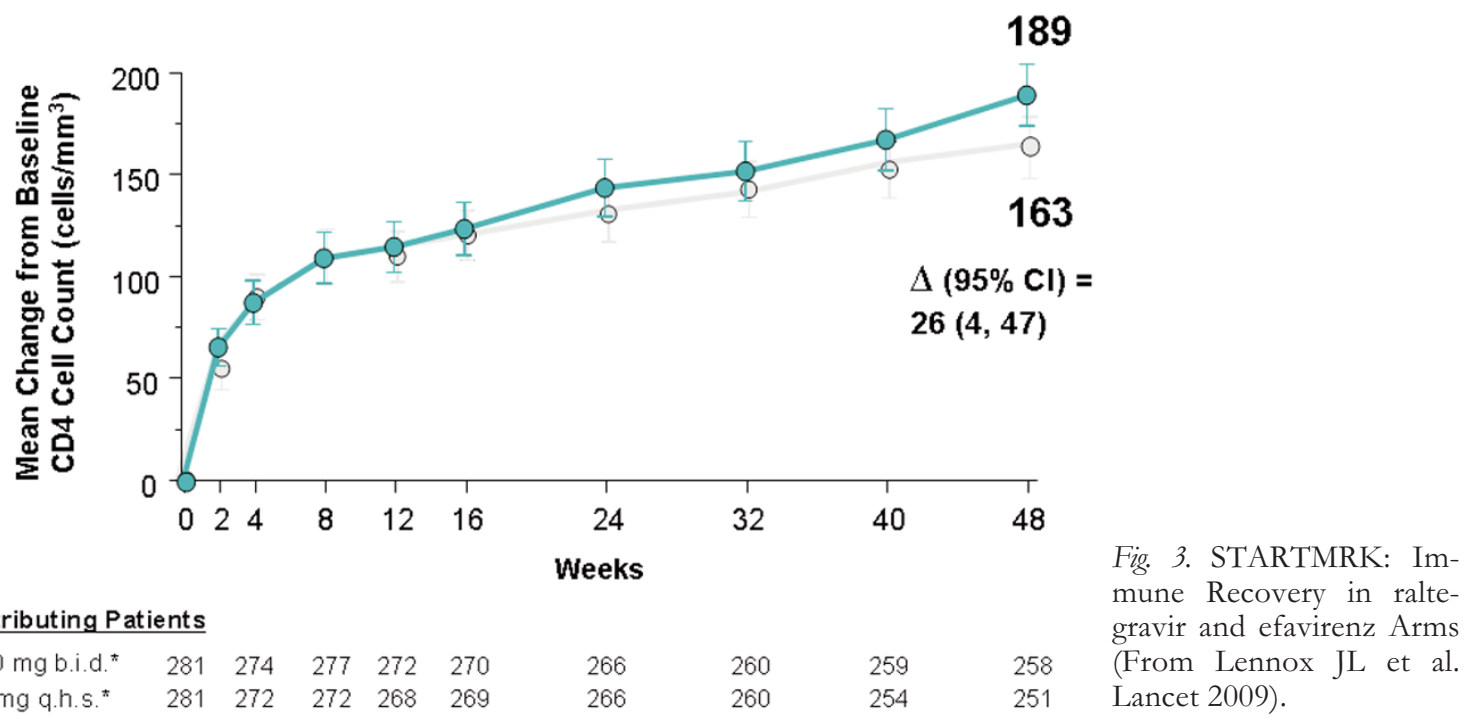

an impact on the magnitude of viral reservoirs, especially since studies have shown that the amount of proviral DNA declines during HAART [20] and in vitro studies, showed how raltegravir and other integrase inhibitors increased the amount of nonintegrated DNA [21]. Perhaps their specific mechanism of action reduces the relative proportion of integrated DNA as compared to the unintegrated counterpart, reducing in other terms the magnitude of infected cells.

Another interesting finding of STARTMRK trial, was the greater CD4+ cell increase at week 48 in the raltegravir arm compared to the efavirenz arm (Fig. 3) and greater increase in CD4+ at week 48 was observed for patients with 100000 copies HIV-1 RNA or less at baseline. This was not observed in the phase II trial, but interestingly was found in patients who switched from enfuvirtide to raltegravir in a simplification strategy study. The potential raltegravir specific role in immune recovery, needs certainly further investigation.
The STARTMRK study also showed how CD4+ at baseline are a prognostic indicator of virologic outcome: a greater proportion of patients starting therapy at higher CD4 count $\left(>200\right.$ cells $\left./ \mathrm{mm}^{3}\right)$ achieved virologic success at week 48 in both raltegravir and efavirenz arms when compared to patients starting at lower CD $4+$ count $\left(<50\right.$ cells $\left./ \mathrm{mm}^{3}\right)$ (Table 1$)$. These data will certainly reinforce the discussion on when to star antiretroviral treatment giving support of an early initiation.

\section{SAFETY AND TOLERABILITY}

Raltegravir is generally a well tolerated drug. The fact that this compound is not metabolized via the cytochrome P450 [22,23] is certainly a major contributor to the low rate of adverse events experienced by subjects in raltegravir studies.

In pharmacokinetic studies the agent was safe up to doses of $1600 \mathrm{mg}$ daily for up to ten days and no sub- 
Table 1. Prognostic indicators of achievement of HIV-1 RNA $<50$ copies $/ \mathrm{mL}$ at week 48.

\begin{tabular}{|c|c|c|c|c|c|}
\hline \multirow[b]{2}{*}{ Overall } & \multicolumn{2}{|c|}{ Raltegravir Group (A) } & \multicolumn{2}{|c|}{ Efavirenz Group (B) } & \multirow[t]{2}{*}{ Difference (A-B) } \\
\hline & $\begin{array}{l}\mathrm{n} / \mathrm{N} \\
241 / 263\end{array}$ & $\begin{array}{l}\text { Response rate } \\
91.6 \%(87.6-94.7)\end{array}$ & $\begin{array}{l}\mathrm{n} / \mathrm{N} \\
230 / 258\end{array}$ & $\begin{array}{l}\text { Response rate } \\
89.1(84.7-92.7)\end{array}$ & \\
\hline \multicolumn{6}{|c|}{ Baseline Plasma HIV-1 RNA (copies/mL) } \\
\hline$\leq 100000$ & $111 / 120$ & $92.5 \%(86.2-96.5)$ & $114 / 128$ & $89.1 \%(82.3-93.9)$ & $3.4 \%(-4.1-11)$ \\
\hline$>100000$ & $130 / 143$ & $90.9 \%(85-95.1)$ & $116 / 130$ & $89.2 \%(82.6-94)$ & $1.7 \%(-5.6-9.2)$ \\
\hline \multicolumn{6}{|c|}{ Baseline CD4 count cells $/ \mu \mathrm{L}$} \\
\hline$\leq 50$ & $21 / 25$ & $84 \%(63.9-95.5)$ & $24 / 28$ & $85.7 \%(67.3-96.0)$ & $-1.7 \%(-23.0-18.7)$ \\
\hline$>50 \leq 200$ & $85 / 95$ & $89.5 \%(81.5-94.8)$ & $83 / 97$ & $85.6 \%(77.0-91.9)$ & $3.9 \%(-5.7-13.7)$ \\
\hline$>200$ & $135 / 143$ & $94.4 \%(89.3-97.6)$ & $122 / 132$ & $92.4 \%(86.5-96.3)$ & $2.0(-4.1-8.5)$ \\
\hline Unknown & 0 & NA & $1 / 1$ & $100 \%(2.5-100)$ & NA \\
\hline \multicolumn{6}{|c|}{ HIV-1 Subtype } \\
\hline Clade B & $186 / 206$ & $90.3 \%(85.4-94.0)$ & $185 / 209$ & $88.5 \%(83.492 .5)$ & $1.8 \%(-4.37 .9)$ \\
\hline Clade Non-B & $52 / 54$ & $96.3 \%(87.3-99.5)$ & $40 / 44$ & $90.9 \%(78.3-97.5)$ & $5.4 \%(-4.9-18.0)$ \\
\hline Unknown & $3 / 3$ & $100.0 \%(29.2-100.0)$ & $5 / 5$ & $100.0 \%(47.8-100.0)$ & $0.0 \%(-59.4-46.8)$ \\
\hline
\end{tabular}

(From Lennox JL et al. Lancet 2009)

ject discontinued the drug due to side effects. The most common drug-related adverse events were headache and fatigue, which were usually transient and mild to moderate in intensity [23]. Results from the first phase III international trials in experienced patients, showed a favourable side-effect profile of raltegravir [13]. No significant difference was found between the raltegravir and the placebo arm for either clinical or laboratory adverse event. The most common drug-related clinical adverse events were diarrhea, nausea and headache in both arms. The most common drug-related laboratory abnormalities were increase in serum lipids, aminotransferase and creatinine. In these studies slightly more patients in the raltegravir arm had fasting cholesterol $>300 \mathrm{mg} / \mathrm{dL}$ and fasting triglycerides $>750 \mathrm{mg} / \mathrm{dL}$. Also higher incidence of serum pancreatic lipase greater than twice the upper limit of normal and serum creatine phosphokinase elevation to levels greater than 10 times the upper limit of normal was observed in the raltegravir arm. Although this difference was not statistically different from the placebo arm, the drug package insert suggests caution in using raltegravir association of other medications known to cause creatine phosphokinase elevation.

In studies on naïve patients when raltegravir was compared to efavirenz $[14,15]$, patients on the sperimental arm experienced less neurologic and psychiatric symptoms related to drug neurotoxicity. In protocol 004 by week 8 , double the number of patients in the efavirenz arm (42\%) experienced neuropsychiatric side effects than patients in the raltegravir arm $(21 \%)$. This trend remained the same throughout the entire study. At week 144 this proportion was 35\% in the raltegravir arm vs $61 \%$ in the efavirenz arm [18]. One of the phase III STARTMRK trial secondary endpoints was superiority of raltegravir in causing fewer central nervous system (CNS) side effects compared to efavirenz. Analysis at week 8 showed that 10\% $(n=29)$ and $18 \%$
( $\mathrm{n}=50$ ) patients in the raltegravir and efavirenz arm respectively had experienced at least one CNS-related adverse event $(p=0.02)$. At week 48 CNS side effects had a cumulative incidence of $14 \%$ in the raltegravir $\operatorname{arm}$ vs $23 \%$ in the efavirenz arm $(p=0.004)$.

Pancreatic toxicity was more frequent in the raltegravir group in protocol 004 as well: after 96 weeks 4 $(2.5 \%)$ and $3(1.3 \%)$ patients and had pancreatic amylase greater than twice the upper limit of normal and pancreatic lipase greater than three times the upper limit of normal respectively, while neither was observed in any patients on efavirenz. Creatinine phosphokinase elevation was more frequent in raltegravir arm $(10 / 160$, $6.3 \%)$ as compared to efavirenz arm $(1 / 38,2.6 \%)$. No cases were associated with clinical adverse experience such as myopathy, myositis or rhabdomyolysis and none required permanent discontinuationof the study therapy. Six out of ten cases were considered related to strenuous exercise. On patient temporarily interrupted raltegravir due to $\mathrm{CK}$ Grade 4 elevation which, however did not reoccur with rechallenge.

Raltegravir also seems to have a safe lipid profile, in the BENCHMRK studies [13] slightly more patients in the raltegravir arm had fasting cholesterol $>300$ $\mathrm{mg} / \mathrm{dL}$ and fasting triglycerides $>750 \mathrm{mg} / \mathrm{dL}$ in the raltegravir arm. Opposite results were observed in naïve patients: higher percentage of patients with fasting total cholesterol $>300 \mathrm{mg} / \mathrm{dL}$, fasting LDL cholesterol $>190 \mathrm{mg} / \mathrm{dL}$ and fasting triglycerides $>750$ $\mathrm{mg} / \mathrm{dL}$ were observed in the efavirenz arm both at 96 and at 144 weeks of phase II trials as well as at 48 weeks in the phase III STARTMRK trial. The latter study also found a small decline in triglycerides concentration in the raltegravir arm at week 48 compared to baseline. In addition of the patients who were not on lipid lowering agents at baseline, a higher number of those who had to start such treatment were in the efavirenz $(\mathrm{n}=11 / 14)$ than in raltegravir arm $(3 / 14)$. 
Table 2. Comprehensive analysis of cancer risk in raltegravir trials.

\begin{tabular}{|c|c|c|c|c|c|}
\hline & \multicolumn{2}{|c|}{ Raltegravir } & \multicolumn{2}{|c|}{ Comparator } & \multirow[t]{2}{*}{ Relative Risk } \\
\hline & & $\begin{array}{c}\text { Cases/Year at } \\
\text { Risk }\end{array}$ & & $\begin{array}{c}\text { Cases/Year at } \\
\text { Risk }\end{array}$ & \\
\hline & $\mathrm{N}$ & (Rate) & $\mathrm{N}$ & & $(95 \% \mathrm{CI})$ \\
\hline Overall & 1039 & $\begin{array}{c}29 / 1722 \\
(1.68)\end{array}$ & 605 & $\begin{array}{c}17 / 159 \\
(2.24)\end{array}$ & $\begin{array}{c}0.75 \\
(0.40-1.46)\end{array}$ \\
\hline \multicolumn{6}{|c|}{ Treatment experienced patients } \\
\hline Protocol 005 & 133 & $0 / 100(0.00)$ & 45 & $0 / 23(0.00)$ & \\
\hline BENCHMRK-1 & 232 & $12 / 417(2.88)$ & 118 & $2 / 133(1.50)$ & \\
\hline BENCHMRK-2 & 230 & $12 / 384(3.12)$ & 199 & $5 / 131(3.81)$ & \\
\hline \multicolumn{6}{|c|}{ Treatment naïve patients } \\
\hline PROTOCOL 004 & 163 & $4 / 429(0.93)$ & 41 & $1 / 100(1.00)$ & \\
\hline STARTMRK & 281 & $1 / 392(0.26)$ & 282 & $9 / 371(2.42)$ & \\
\hline
\end{tabular}

(From Cooper et al. 2009)

Also, twenty one patients were already on lipid-lowering treatment at baseline, and the number of these patients who increased the medication dose or had to add another medication, was the same in the two arms $(n=4)$.

Data from the BENCHMRK studies raised a worrisome concern when a higher incidence of malignancies (recurrent, progression or new diagnosis of cancer) was found in the raltegravir arm $(16 / 462,3.5 \%)$, compared to the placebo arm $(4 / 237,1.7 \%)$ showing a relative risk of 1.5 (95\% CI 0.5-6.3). Patients on raltegravir arm had diagnosis of cancer earlier in the study (median = 68 days, IQR 30-118) than patients in placebo arm (median $=285$ days, IQR 246-336). By the time of cancer diagnosis, all but two patients had had a viroimmuonologic response to treatment: HIV-RNA decrease of $1 \log _{10}$ or more or $<400$ copies/mL and CD $4+$ cell increase of 50 cells $/ \mu \mathrm{L}$ or more. Only in three raltegravir patients however, immune reconstitution was considered by the investigators to have possibly contributed to the diagnosis of cancer. Recently a comprehensive analysis of cancer risk in raltegravir trials, including data from the phase II and III studies, both naïve and experienced patients, showed a malignancy rate of $1.7 / 100$ patients years in raltegravir arms compared to a rate of $2.2 / 100$ patients years in the comparator arms, resulting in a relative risk of 0.8 (95\% CI 0.4-1.5) (Table 1) [24]. Considering naïve patients only, the malignancy incidence rate was $0.6 / 100$ patients years in the raltegravir arms and 2.1/100 patients years in the comparator arms. In the STARTMRK trial, one new or recurrent cancer $(<1 \%$, Kaposi's sarcoma) was diagnosed in the raltegravir arm while nine $(3 \%)$ cancers were diagnosed in the efavirenz arm (Kaposi's sarcoma $\mathrm{n}=6$, B-cell lymphoma $\mathrm{n}=1$, squamous cell carcinoma of the anus $n$ $=1$ and bone cancer $\mathrm{n}=1$ ). Fortunately the malignancy trend in naive patients seems to have overturn the first concerns arose by results in experienced patients, and overall cancer rate in raltegravir receiving patients seems not to differ from standard of care agents.

\section{RESISTANCE}

In the BENCHMRK studies $23 \%$ of patients in the raltegravir arm experienced virolgic failure at week 48 and $43 \%$ at week 96 [13]. Data form the use of raltegravir in drug experienced patients showed that resistance to raltegravir usually emerge following one of three pathways each involving mutations at one of three sites in association with other mutations [25]. The N155 pathway is characterized by the N155H mutation often in association with the E92Q. It is usually the first resistance mutation pattern observed in patients failing a raltegravir based regimen. After several weeks under pharmacologic pressure it is substitute by mutations arising from other pathways. The second pathway is characterized by mutations at site Q148, which can mutate to $\mathrm{H}, \mathrm{R}$ or $\mathrm{K}$, most often followed by the G140S mutation. This pathway emerge usually later in patients failing a raltegravir regimen. The third pathway emerges with the Y143R mutation, usually followed by T97A E92Q. Interestingly clonal analysis showed that mutations belonging to different pathways can coexist in different genomes in the viral population of an infected subject and can coevolve in parallel [26]. These resistance pathways, however, seem to be mutually exclusive, they were never found in the same viral genome in the same patient. Recently, data showing a higher and broader selective advantage profile as a function of drug concentration, for the N155H mutants in comparison to Q148H mutant seemed to explain the longitudinal trends in the resistance mutation pathways [27]. These pathways were observed in patients enrolled in clinical trials, data from the use of raltegravir in clinical settings might differ from the strict rules of these pathways. Of notice, preliminary in vitro data and results from clinical trials show that resistance to raltegravir can be achieved with one mutation only, suggesting a relatively low genetic barrier for this drug. In protocol 004 virologic failure occurred in $4 \%$ $(6 / 160)$ of patients in the raltegravir group and 5\% 
$(2 / 38)$ patients in the efavirenz group at week $96[17]$. At week 144 other 2 patients in the raltegravir arm and 1 patient in the efavrenz arm experienced virologic failure leading to a failure rate of $5 \%$ and $8 \%$ respectively. Five of the eight raltegravir-treated patients who failed had resistance mutations in their integrase gene, the most common being $\mathrm{N} 115 \mathrm{H}(\mathrm{n}=2)$. All three patients in the efavirenz arm had one or more resistance mutations to the drug at the time of failure. The most common NRTI mutations in both groups were M184V $(\mathrm{n}=5)$ and K65R $(\mathrm{n}=4)$ [18]. In STARTMRK trial $10 \%(27 / 280)$ patients in the raltegravir arm and $14 \%$ $(39 / 281)$ patients in the efavirenz arm experienced virologic failure at week 48. In the raltegravir arm, of the nine patients with HIV-RNA > 400 copies, which allowed for genotypic analysis, four did not show any mutation in their integrase gene, one did not have amplification results and four patients had known integrase resistance mutations. Of the latter group, two patients showed the Q148H/R + G140S pathway, one patient showed the Y143R mutation only and one patient showed the $\mathrm{Y} 143 \mathrm{Y} / \mathrm{H}$ mixture in association with L74L/M + E92Q + T97T/A mutations [14]. Although longitudinal data on previously naïve patients failing raltegravir-based regimens are not available and will probably be difficult to obtain, the evolution of resistance pathways seems somehow different from what observed in drug-experienced patients.

\section{EXPERT OPINION FOR THE UsE IN TREATMENT Naive Patients}

Raltegravir is the first integrase inhibitor to be approved for the use in the treatment of HIV infection. Based on results of recent international trials, the US FDA has recently approved its use also for naïve patients. Although not yet recommended by international guidelines, a great increase in the use of this compound as the first line treatment is certainly predictable. In naïve patients, raltegravir has demonstrated mid- and long-term efficacy in reducing HIV-RNA to $<50$ copies, comparable to that of other recommended first line agents $[14,15,18]$. In the short-term in addition, raltegravir showed a faster viral decay compared to efavirenz. The reason and meaning of this is still to be definitely elucidated, although recently, mathematical models confirmed how in general the later an inhibitor acts in HIV life cycle, the more rapid the decay in viremia [28]. Also, interesting models were developed to study the specific contribution of an integrase inhibitor, suggesting a reduced second phase decline of HIV-RNA in patients treated with this compound [19]. The authors suggested that this raltegravir effect might have an impact in reducing the time needed for clearance of infection. This hypothesis might have been far too optimistic given the subsequent demonstration of a third and fourth phase of viral decay after a longterm viral suppression, shown to be due to cells that had a half-life not different from infinite, mirroring long-lived chronically infected cells [29]. Also some recent HAART intensification studies of patients suppressed for a long time, showed no additional viral decay when raltegravir was added to their regimen, reinforcing the small role antiretrovirals have on latently infected cells [30-32]. Although the possibility of viral clearance after infection might be still unrealistic, the additional specific effect of integrase inhibition on viral decay might have an impact on the magnitude of viral reservoirs, especially when used when this pool constitutes, i.e. during acute-early infection [33, 34]. Proviral DNA decay during HAART [20], and patients starting antiretroviral treatment during acute or recent infection have significantly smaller amount of viral DNA after 36 weeks of treatment than patients starting HAART during chronic infection [35]. The relative proportion of integrated DNA, however, seems to be higher on treatment compared to pretherapy, when patients are on standard HAART [20]. The specific effect of integrase inhibitors observed in vitro of increasing unintegrated DNA [21], might reflect a reduction in the viral integration in newly infected cells, this reducing the spread of infection. This specific effect might be of extreme relevance during acute infection; studies exploring the effect of raltegravir (in association with other compounds) during primary infection are ongoing [36]. Clinically, the importance of this faster initial viral decay lays in the correlation between viral dynamic after initiation of antiretroviral treatment and longterm success [37-39]. These data, together with efficacy and tolerability profiles of new antiretroviral therapies, the prognostic role of CD4+ count at baseline $[14,40-43]$ and cohort studies showing a clinical benefit of early treatment of HIV infection [44] reinvigorate the ongoing discussion of when to start treatment for asymptomatic HIV infection supporting the early treatment option. Thanks to the faster viral decay raltegravir might also find a place in post-exposure profilaxis; an integrase inhibitor, perhaps in association with entry inhibitors, might reduce the spreading of cell infection and the magnitude of viral reservoirs.

In chronically infected patients, long-term success is also assured by an effective regimen. Transmitted drug-resistance is a critical issue in choosing first line regimen and its prevalence is still significant in developed countries and in Europe as well [5, 45, 46]. Efavirenz-based therapy is one of the preferred the first line regimen, thanks to its long term efficacy and the recent availability of a fixed-dose combination with tenofovir and emtricitabine in a single pill daily. Transmitted resistance, however, might jeopardize the effectiveness of this combination, being NNRTI and NRTI resistance mutation the most common transmitted resistance mutations. HIV genotyping before initiation of antiretroviral treatment is recommended by international guidelines, nevertheless there are clinical settings in which this guideline is not fully implemented. In addition wild type virus might overgrow resistant variants in the absence of drug pressure; resistant viral strains however, will re-emerge in the presence of a selective drug pressure, leading to virologic failure. Raltegravir, being a new class compound, might be a choice to overcome transmitted drug resistance.

Although found in the phase III trial only and not confirmed by studies with longer follow-up, the greater increase in CD4+ cells compared to efavirenz, is certainly an additional reason that might favor the use of raltegravir in treatment naïve patients, especially for certain categories of patients as late presenters. 
A greater viral decay and increase in CD4+ T cells, raises the issue of Immune Reconstitution Syndrome for patients starting at low levels of CD4+ cells. Overall incidence of the syndrome in trials on naïve patients is not clear, but does not appear to be high. Given the importance of short-term side effects on adherence to treatment, the lower incidence of CNS side effects in the raltegravir arm compared to efavirenz will certainly impact on the choice of first line regimen, also considering that the majority of failures of first line treatment happen in the firs weeks of treatment where acute side effects play a major role. Also, the safe lipid and metabolic profile might make raltegravir a preferred choice over PI-based regimens in light of the long-term toxicities of HAART especially for patients who already have other risk factors [47]. Associations of raltegravir and other recently approved compounds proved to be effective in multidrug resistant patients, showing also a safe metabolic profile. Similar combinations have not been studied in naive patients yet, but they might offer interesting options to avoid long-term toxicities of HAART. Trials exploring these strategies are ongoing [48].

Integrase inhibitors specific long-term toxicities are not completely defined yet, as the compounds have not been available for longer than 5 years, and have been used on a large scale only recently. Initial concerns on higher malignancy incidence in raltegravir-receiving patients seem to be withdrawn after an extensive analysis on all raltegravir trials participants. Data on the possible impact of integrase inhibitors on carcinogenesis are not available and additional studies on this matter are certainly needed.

Raltegravir has already found its place in the treatment of multidrug experienced patients, and is a promising option for naive patients too. A great debate is ongoing whether its best role would be in the salvage treatment, given its efficacy in patients with limited therapeutic options and the possibility of constructing a fully active regimens in association with other new class compounds; or its best place being earlier in the treatment history of a patients, thanks to its favorable tolerability and safety profile. A large use of this new compound in naïve patients in predictable in the near future, only time and experience will tell us whether raltegravir will maintain its promises in the long run.

\section{REFERENCES}

1. Palella FJ,Jr, Delaney KM, Moorman AC, Loveless MO, Fuhrer J, Satten GA, et al. Declining morbidity and mortality among patients with advanced human immunodeficiency virus infection. HIV Outpatient Study Investigators. N Engl J Med 1998; 338: 853.

2. Mocroft A, Vella S, Benfield TL, Chiesi A, Miller V, Gargalianos $\mathrm{P}$, et al. Changing patterns of mortality across Europe in patients infected with HIV-1. EuroSIDA Study Group. Lancet 1998; 352: 1725.

3. Buchbinder SP, Mehrotra DV, Duerr A, Fitzgerald DW, Mogg R, Li D, et al. Efficacy assessment of a cell-mediated immunity HIV-1 vaccine (the Step Study): a doubleblind, randomised, placebo-controlled, test-of-concept trial. Lancet 2008; 372: 1881

4. Robb ML. Failure of the Merck HIV vaccine: an uncertain step forward. Lancet 2008; 372: 1857.
5. Wensing AM, van de Vijver DA, Angarano G, Asjo B, Balotta C, Boeri E, et al. Prevalence of drug-resistant HIV-1 variants in untreated individuals in Europe: implications for clinical management. J Infect Dis 2005; 192: 958.

6. Wheeler W, Mahle K, Bodnar U, et al. Antiretroviral drug-resistance mutations and subtypes in drug-naive persons newly diagnosed with HIV-1 infection, US, March 2003 to October 2006. 14th conference on retroviruses and opportunistic Infections, Los Angeles, CA, February 25-28, 2007. 2007.

7. DHHS Panel on Antiretroviral Guidelines for Adults and Adolescents - A Working Group of the Office of AIDS Research Advisory Council (OARAC). Guidelines for the Use of Antiretroviral Agents in HIV-1-Infected Adults and Adolescents. 2008.

8. Clumeck N, Pozniak A, Raffi F for the European AIDS Clinical Society (EACS) Guidelines for the clinical management and treatment of HIV infected adults in Europe. HIV Med. 2008 Feb;9(2):65-71.

9. Chesney MA. The elusive gold standard. Future perspectives for HIV adherence assessment and intervention. J Acquir Immune Defic Syndr 2006; 43 Suppl 1: S149.

10. O'Brien ME, Clark RA, Besch CL, Myers L, Kissinger P. Patterns and correlates of discontinuation of the initial HAART regimen in an urban outpatient cohort. J Acquir Immune Defic Syndr 2003; 34: 407.

11. Lazzarin A, Campbell T, Clotet B, Johnson M, Katlama C, Moll A, et al. Efficacy and safety of TMC125 (etravirine) in treatment-experienced HIV-1-infected patients in DUET-2: 24-week results from a randomised, doubleblind, placebo-controlled trial. Lancet 2007; 370: 39.

12. Gulick RM, Lalezari J, Goodrich J, Clumeck N, DeJesus E, Horban A, et al. Maraviroc for previously treated patients with R5 HIV-1 infection. N Engl J Med 2008; 359: 1429.

13. Steigbigel RT, Cooper DA, Kumar PN, Eron JE, Schechter M, Markowitz M, et al. Raltegravir with optimized background therapy for resistant HIV-1 infection. N Engl J Med 2008; 359: 339.

14. Lennox JL, Dejesus E, Lazzarin A, Pollard RB, Madruga JV, Berger DS, et al. Safety and efficacy of raltegravirbased versus efavirenz-based combination therapy in treatment-naive patients with HIV-1 infection: a multicentre, double-blind randomised controlled trial. Lancet. 2009 Sep 5;374(9692):796-806.

15. Markowitz M, Nguyen BY, Gotuzzo E, Mendo F, Ratanasuwan W, Kovacs C, et al. Rapid and durable antiretroviral effect of the HIV-1 Integrase inhibitor raltegravir as part of combination therapy in treatment-naive patients with HIV-1 infection: results of a 48-week controlled study. J Acquir Immune Defic Syndr 2007; 46: 125.

16. Markowitz M, Morales-Ramirez JO, Nguyen BY, Kovacs CM, Steigbigel RT, Cooper DA, et al. Antiretroviral activity, pharmacokinetics, and tolerability of MK-0518, a novel inhibitor of HIV-1 integrase, dosed as monotherapy for 10 days in treatment-naive HIV-1-infected individuals. J Acquir Immune Defic Syndr 2006; 43: 509.

17. Markowitz M, Nguyen BY, Gotuzzo E, Mendo F, Ratanasuwan W, Kovacs C, et al. Sustained antiretroviral effect of raltegravir after 96 weeks of combination therapy in treatment-naive patients with HIV-1 infection. J Acquir Immune Defic Syndr 2009; 52: 350-356

18. Gotuzzo E, Nguyen BY, Markowitz M, Mendo F, Ratanasuwan W, Kovacs C, et al. Sustained Antiretroviral Efficacy of Raltegravir as part of Combination ART in Treatment-Naive HIV-1 infected patients: 144-week data. 5th IAS conference on HIV Pathogenesis, Treatment and Prevention. Cape Town,July, 19-22 2009. Abstract \#A-1550079-01895. 2009. 
19. Murray JM, Emery S, Kelleher AD, Law M, Chen J, Hazuda DJ, et al. Antiretroviral therapy with the integrase inhibitor raltegravir alters decay kinetics of HIV, significantly reducing the second phase. AIDS 2007; 21: 2315.

20. Koelsch KK, Liu L, Haubrich R, May S, Havlir D, Gunthard HF, et al. Dynamics of total, linear nonintegrated, and integrated HIV-1 DNA in vivo and in vitro. J Infect Dis 2008; 197: 411

21. Goffinet C, Allespach I, Oberbremer L, Golden PL, Foster SA, Johns BA, et al. Pharmacovirological impact of an integrase inhibitor on human immunodeficiency virus type 1 cDNA species in vivo. J Virol 2009; 83: 7706.

22. Merck and Co. I. Isentress (raltegravir) package insert. 2007.

23. Iwamoto M, Wenning LA, Petry AS, Laethem M, De Smet M, Kost JT, et al. Safety, tolerability, and pharmacokinetics of raltegravir after single and multiple doses in healthy subjects. Clin Pharmacol Ther 2008; 83: 293.

24. Cooper DA, Steigbigel RT, Lennox JL, Grinsztejn B, Markowitz M, Sklar P, et al. Review of Cancer Incidence in Raltegravir (RAL) Clinical Trials.16th Conference on Retroviruses and Opportunistic Infections. Montreal, Canada February 8-11, 2009. Abstract R-106. 2009; Abstract R-106.

25. Cooper DA, Steigbigel RT, Gatell JM, Rockstroh JK, Katlama C, Yeni P, et al. Subgroup and resistance analyses of raltegravir for resistant HIV-1 infection. N Engl J Med 2008; 359: 355.

26. Fransen S, Karmochkine M, Huang W, Weiss L, Petropoulos CJ, Charpentier C. Longitudinal analysis of raltegravir susceptibility and integrase replication capacity of HIV-1 during virologic failure. Antimicrob Agents Chemother 2009.

27. Quercia R, Dam E, Perez-Bercoff D, Clavel F. Selective advantage profile of HIV-1 integrase mutants explain in vivo evolution of raltegravir resistance genotypes. J Virol 2009.

28. Sedaghat AR, Dinoso JB, Shen L, Wilke CO, Siliciano RF. Decay dynamics of HIV-1 depend on the inhibited stages of the viral life cycle. Proc Natl Acad Sci U S A 2008; 105 : 4832.

29. Palmer S, Maldarelli F, Wiegand A, Bernstein B, Hanna GJ, Brun SC, et al. Low-level viremia persists for at least 7 years in patients on suppressive antiretroviral therapy. Proc Natl Acad Sci U S A 2008; 105: 3879.

30. Dinoso JB, Kim SY, Wiegand AM, Palmer SE, Gange SJ, Cranmer L, et al. Treatment intensification does not reduce residual HIV-1 viremia in patients on highly active antiretroviral therapy. Proc Natl Acad Sci U S A 2009; 106: 9403.

31. Jones J, McMahon D, Wiegand A, Kearney M, Palmer S, McNulty S, et al. No Decrese in Residual Viremia during Raltegravir Intensification in Patients on Standard ARV. 16th conference on retroviruses and Opportunistic Infections. Montreal, Canada. February 8-11, 2009. Abstract 432b. 2009.

32. Gandhi R, Zheng S, Bosch R, Chan E, Margolis D, Read $\mathrm{S}$, et al. Raltegravir (RAL) intensification does not reduce low-level residual viremia in HIV-1-infected patients on antiretroviral therapy (ART): results from ACTG A5244. 5th IAS Conference on HIV Pathogenesis, treatment and Prevention. Cape Town, South Africa July 19-22, 2009. 2009.

33. Finzi D, Hermankova M, Pierson T, Carruth LM, Buck C, Chaisson RE, et al. Identification of a reservoir for HIV-1 in patients on highly active antiretroviral therapy. Science 1997; 278: 1295.

34. Finzi D, Blankson J, Siliciano JD, Margolick JB, Chadwick $\mathrm{K}$, Pierson $\mathrm{T}$, et al. Latent infection of $\mathrm{CD} 4+\mathrm{T}$ cells provides a mechanism for lifelong persistence of HIV-1, even in patients on effective combination therapy. Nat Med 1999; 5: 512.

35. Pires A, Hardy G, Gazzard B, Gotch F, Imami N. Initiation of antiretroviral therapy during recent HIV-1 infection results in lower residual viral reservoirs. J Acquir Immune Defic Syndr 2004; 36: 783.

36. South East Asia Research Collaboration with Hawaii. Antiretroviral Therapy for Acute HIV Infection (AAHIV). ClinicalTrials.gov Identifier:NCT00796263. 2009.

37. Polis MA, Sidorov IA, Yoder C, Jankelevich S, Metcalf J, Mueller BU, et al. Correlation between reduction in plasma HIV-1 RNA concentration 1 week after start of antiretroviral treatment and longer-term efficacy. Lancet 2001; 358: 1760.

38. Louie M, Hogan C, Di Mascio M, Hurley A, Simon V, Rooney J, et al. Determining the relative efficacy of highly active antiretroviral therapy. J Infect Dis 2003; 187: 896.

39. Kuritzkes DR, Ribaudo HJ, Squires KE, Koletar SL, Santana J, Riddler SA, et al. Plasma HIV-1 RNA dynamics in antiretroviral-naive subjects receiving either triple-nucleoside or efavirenz-containing regimens: ACTG A5166s. J Infect Dis 2007; 195: 1169.

40. Walmsley S, Bernstein B, King M, Arribas J, Beall G, Ruane $P$, et al. Lopinavir-ritonavir versus nelfinavir for the initial treatment of HIV infection. N Engl J Med 2002; 346: 2039.

41. Murphy RL, da Silva BA, Hicks CB, Eron JJ, Gulick RM, Thompson MA, et al. Seven-year efficacy of a lopinavir/ ritonavir-based regimen in antiretroviral-naive HIV-1-infected patients. HIV Clin Trials 2008; 9: 1.

42. Molina JM, Andrade-Villanueva J, Echevarria J, Chetchotisakd P, Corral J, David N, et al. Once-daily atazanavir/ ritonavir versus twice-daily lopinavir/ritonavir, each in combination with tenofovir and emtricitabine, for management of antiretroviral-naive HIV-1-infected patients: 48 week efficacy and safety results of the CASTLE study. Lancet 2008; 372: 646.

43. Mills AM, Nelson M, Jayaweera D, Ruxrungtham K, Cassetti I, Girard PM, et al. Once-daily darunavir/ritonavir vs. lopinavir/ritonavir in treatment-naive, HIV-1-infected patients: 96-week analysis. AIDS 2009; 23: 1679.

44. Kitahata MM, Gange SJ, Abraham AG, Merriman B, Saag MS, Justice AC, et al. Effect of early versus deferred antiretroviral therapy for HIV on survival. N Engl J Med 2009; 360: 1815.

45. Alteri C, Svicher V, Gori C, D'Arrigo R, Ciccozzi M, Ceccherini-Silberstein F, et al. Characterization of the patterns of drug-resistance mutations in newly diagnosed HIV-1 infected patients naive to the antiretroviral drugs. BMC Infect Dis 2009; 9: 111.

46. Bracciale L, Colafigli M, Zazzi M, Corsi P, Meraviglia P, Micheli V, et al. Prevalence of transmitted HIV-1 drug resistance in HIV-1-infected patients in Italy: evolution over 12 years and predictors. J Antimicrob Chemother 2009; 64: 607.

47. DAD Study Group, Friis-Moller N, Reiss P, Sabin CA, Weber R, Monforte A, et al. Class of antiretroviral drugs and the risk of myocardial infarction. N Engl J Med 2007; 356: 1723.

48. University of Miami. Raltegravir + Lopinavir/Ritonavir or Emtricitabine/Tenofovir for HIV Treatment Naive Subjects. ClinicalTrials.gov Identifier: NCT00654147. 2009.

\section{Address for correspondence:}

Adriano Lazzarin

Department of Infectious Diseases

San Raffaele Scientific Institute

Via Olgettina 60

20132 Milano

Italy

E-mail: lazzarin.adriano@hsr.it 